\section{Eficacia a largo plazo de la desensibilización a aspirina en enfermedad respiratoria exacerbada por aspirina. Revisión de 2 casos clínicos}

Julio César Cambray-Gutiérrez, ${ }^{1}$ Ulises Noel García-Ramírez, ${ }^{2}$ Leonel Gerardo Del Rivero-Hernández, ${ }^{1}$ Sean Alejandro Lozano-Martínez, ${ }^{2}$ Patricia LópezPérez, ${ }^{1}$ Aurora Chávez-García ${ }^{1}$

\section{Resumen}

ANTECEDENTES: la prevalencia de enfermedad respiratoria exacerbada por aspirina es de $7 \%$ en pacientes asmáticos y se incrementa, incluso, a $14 \%$ en pacientes con asma de difícil control. El tratamiento incluye la prescripción de inhibidores de los receptores de leucotrienos, esteroides intranasales, polipectomías, tratamiento del asma según su severidad y evitar los antiinflamatorios no esteroides. En algunos pacientes es necesario realizar el protocolo de desensibilización a la aspirina.

CASOS CLÍNICOS: se describen 2 mujeres con diagnóstico de enfermedad respiratoria exacerbada por la administración de aspirina, con escaso control de los cuadros de asma y a quienes fue necesario realizar múltiples polipectomías, a pesar del manejo farmacológico óptimo. Se llevó a cabo protocolo de desensibilización a aspirina (AAS); la respuesta fue positiva. Después de cuatro años, las pacientes presentan adecuado control del asma, con una dosis de mantenimiento de AAS de 150 mg/ día y no han requerido polipectomías.

PALABRAS CLAVE: enfermedad respiratoria exacerbada por aspirina, desensibilización.

Rev Alerg Méx 2016 Apr-Jun;63(2):207-212.

\section{Long-term efficacy of aspirin desensitization in aspirin-exacerbated respiratory disease. Review of two clinical cases}

Julio César Cambray-Gutiérrez, ${ }^{1}$ Ulises Noel García-Ramírez, ${ }^{2}$ Leonel Gerardo Del Rivero-Hernández, ${ }^{1}$ Sean Alejandro Lozano-Martínez, ${ }^{2}$ Patricia LópezPérez, ${ }^{1}$ Aurora Chávez-García ${ }^{1}$

\section{Abstract}

BACKGROUND: The aspirin exacerbated respiratory disease (AERD) shows a prevalence of $7 \%$ among asthmatics and increases to $14 \%$ in patients with difficult to control asthma. Treatment includes the use of
${ }^{1}$ Servicio de Alergia e Inmunología Clínica, Hospital de Especialidades, Centro Médico Nacional Siglo XXI, Instituto Mexicano del Seguro Social, Ciudad de México. ${ }^{2}$ Hospital de Especialidades, Centro Médico Nacional del Bajío, Guanajuato.

Instituto Mexicano del Seguro Social, México.

Recibido: 26 de noviembre 2015

Aceptado: 24 de febrero 2016

Correspondencia

Julio César Cambray-Gutiérrez

jcesar_963@hotmail.com

Este artículo debe citarse como

Cambray-Gutiérrez JC, García-Ramírez UN, Del Rivero-Hernández LG, Lozano-Martínez SA, LópezPérez $\mathrm{P}$, Chávez-García A. Eficacia a largo plazo de la desensibilización a aspirina en enfermedad respiratoria exacerbada por aspirina. Revisión de 2 casos clínicos. Rev Alerg Méx. 2016 abr-jun;63(2):207-212. 
inhibitors of leukotriene receptor (), intranasal steroids, polypectomy, asthma management according to the severity and avoid taking nonsteroidal anti-inflammatory drugs (NSAIDs). In some patients it is necessary desensitization protocol to it.

CLINICAL CASES: 2 patients diagnosed with respiratory disease exacerbated by aspirin, with poor asthma control and need for multiple polypectomies, despite optimal pharmacological management, carrying out protocol desensitization to aspirin (AAS) successful, now after 4 years of having carried out, they have adequate asthma control without need for polypectomies with a maintenance dose of aspirin $150 \mathrm{mg} /$ day.

KEYWORDS: Aspirin exacerbated respiratory disease, desensitization.
${ }^{1}$ Servicio de Alergia e Inmunología Clínica, Hospital de Especialidades, Centro Médico Nacional Siglo XXI, Instituto Mexicano del Seguro Social, Ciudad de México.

${ }^{2}$ Hospital de Especialidades, Centro Médico Nacional del Bajío, Guanajuato.

Instituto Mexicano del Seguro Social, México.

\section{Correspondence}

Julio César Cambray-Gutiérrez

jcesar_963@hotmail.com

\section{ANTECEDENTES}

El primer caso de enfermedad respiratoria exacerbada por aspirina fue descrito en 1902, Widal y su grupo fueron los primeros en establecer la asociación entre asma con sensibilidad a la aspirina y poliposis nasal. En 1922 y 1968 Samter y Beers, respectivamente, denominaron la tríada de Samter. ${ }^{1}$

La enfermedad respiratoria exacerbada por aspirina es una enfermedad caracterizada por la coexistencia de asma, rinosinusitis crónica, poliposis nasal y reacciones adversas al ácido acetil salicílico y otros antiinflamatorios no esteroides. Sus síntomas clínicos son: congestión nasal y broncoconstricción, que suelen manifestarse a los 20 minutos a 3 horas posteriores a la ingestión de antiinflamatorios no esteroides. También se conoce como asma sensible a la aspirina o asma con intolerancia a la aspirina. ${ }^{2}$

La enfermedad respiratoria exacerbada por aspirina tiene una prevalencia de $7 \%$ en pacientes asmáticos y se incrementa incluso a $14 \%$ en pacientes con asma de difícil control. Coexiste en $10 \%$ de los pacientes con poliposis nasal y en $9 \%$ de quienes padecen rinosinusitis crónica. ${ }^{3}$

Las reacciones de los pacientes con enfermedad respiratoria exacerbada por aspirina, posteriores a la ingesta de ácido acetilsalicílico, se denominan pseudoalergia debido a que no se encuentran mediadas por IgE. Son generadas por su capacidad de inhibir la ciclooxigenasa tipo 1 (COX-1). En estos pacientes existe una desregulación del metabolismo del ácido araquidónico, con sobreproducción de los cisteinil leucotrienos (cysLTs): LTC $_{4^{\prime}}$ LTD $_{4}$ y $\mathrm{LTE}_{4}$, potentes broncoconstrictores, inductores de la producción de moco, congestión nasal y quimiotaxis de eosinófilos en las vías aéreas; con bloqueo de la producción de broncodilatadores y antiinflamatorios, como; PGE $_{2}$ y lipoxinas, que inducen el desequilibrio entre la producción de mediadores pro y los antiinflamatorios. ${ }^{4}$

La mayoría de los pacientes resulta con rinitis resistente, seguida por rinosinusitis eosinofílica crónica, caracterizada clínicamente por congestión nasal, anosmia y poliposis nasal, y requerimiento de múltiples polipectomías; posteriormente padecen asma, en la mayoría de los casos de difícil control, lo que disminuye su calidad de vida. ${ }^{5}$

El diagnóstico es clínico y basado en el informe de la tomografía computada de senos paranasales; puede corroborarse con la prueba de reto nasal, bronquial u oral para ácido acetilsalicílico. ${ }^{5}$

En la mayor parte de los centros hospitalarios se prefiere la prueba de reto oral, que se inicia con 
la administración de dosis baja de aspirina, de 30 a $41.5 \mathrm{mg}$, duplicando la dosis cada 3 horas hasta alcanzar 325 o $650 \mathrm{mg}$. Como esquema de premedicación se indica montelukast a dosis de $10 \mathrm{mg} /$ día durante tres días antes de su realización o $10 \mathrm{mg}$ cada 12 horas un día antes.

No está indicada la premedicación con antihistamínicos, cromonas, ni la suspensión de broncodilatadores ni esteroides intranasales e inhalados. Algunos autores recomiendan el uso de placebo. ${ }^{6}$

El costo de cada polipectomía en el Instituto Mexicano del Seguro Social (IMSS), informada en el Diario Oficial del IMSS de 2015, es de $55,667.00$ pesos mexicanos, por lo que es un padecimiento con alto impacto socioeconómico. ${ }^{7}$ (Cuadro 1).

\section{CASOS CLÍNICOS}

\section{Caso 1}

Mujer de 56 años de edad, con antecedente de reacciones adversas tipo $\mathrm{B}$ (broncoespasmo) a los antiinflamatorios no esteroides (diclofenaco, naproxeno y metamizol). A los 45 años de edad inició con rinosinusitis crónica moderada-severa, persistente y asma moderada persistente con

Cuadro 1. Costo desglosado derivado de una polipectomía en el IMSS

\begin{tabular}{lc}
\hline Procedimiento & $\begin{array}{c}\text { Costo en hospital de } \\
\text { tercer nivel. (pesos) }\end{array}$ \\
$\begin{array}{lc}\text { Consulta especialista (envío a oto- } \\
\text { rrinolaringología) }\end{array}$ & $1,717$. \\
$\begin{array}{l}\text { Tomografía computarizada de con- } \\
\text { trol. }\end{array}$ & $2,787$. \\
$\begin{array}{l}\text { Renta de equipo por IMSS para } \\
\text { realizar cirugía }\end{array}$ & $13,000$. \\
$\begin{array}{l}\text { Intervención quirúrgica } \\
\text { Un día de hospitalización }\end{array}$ & $31,432$. \\
Total & $6,731$. \\
& $55,667$.
\end{tabular}

múltiples exacerbaciones y con pobre respuesta al tratamiento con fármacos, previamente requirió 12 polipectomías nasales por obstrucción nasal recurrente (1 polipectomía cada 0.9 años); además, padecía anosmia, por lo que fue enviada a nuestro servicio.

Las pruebas epicutáneas con aeroalergenos fueron negativas, se diagnostica enfermedad respiratoria exacerbada por aspirina y el tratamiento se inició con inhibidores de los receptores de leucotrienos y esteroide nasal. El tratamiento se ajustó al paso 4 de la GINA, sin mejoría clínica, por lo que se consideró apta para el protocolo de desensibilización del ácido acetilsalicílico (Cuadro 2).

Esquema de desensibilización (Cuadro 3)

- Día 0 se administraron 4 dosis de placebo con intervalos de 20 minutos, sin síntomas.

- Día 1: se administraron dosis crecientes de aspirina (AAS) con intervalos de 20 minutos,

Cuadro 2. Características de pacientes sometidas a esquema de desensibilización

\begin{tabular}{|c|c|c|}
\hline Variable & Paciente 1 & Paciente 2 \\
\hline Edad & 56 años & 49 años \\
\hline $\begin{array}{l}\text { Antecedente de alergia a } \\
\text { los antiinflamatorios no } \\
\text { esteroides }\end{array}$ & $\begin{array}{c}\text { Sí } \\
\text { (diclfenaco, } \\
\text { naproxeno, } \\
\text { metamizol) }\end{array}$ & $\begin{array}{l}\text { Si. (aspirina, } \\
\text { metamizol) }\end{array}$ \\
\hline $\begin{array}{l}\text { Edad de inicio de los sín- } \\
\text { tomas }\end{array}$ & 45 años. & 29 años \\
\hline $\begin{array}{l}\text { Clasificación del asma se- } \\
\text { gún su severidad }\end{array}$ & $\begin{array}{l}\text { Moderada } \\
\text { persistente }\end{array}$ & $\begin{array}{l}\text { Grave persis- } \\
\text { tente }\end{array}$ \\
\hline Cantidad de polipectomías & 12 & 6 \\
\hline $\begin{array}{l}\text { Pruebas cutáneas a aeroa- } \\
\text { lergenos }\end{array}$ & Negativas & Positivas \\
\hline $\begin{array}{l}\text { Efectos adversos durante la } \\
\text { desensibilización }\end{array}$ & Sí & Sí \\
\hline $\begin{array}{l}\text { Efectos adversos durante el } \\
\text { mantenimiento }\end{array}$ & No & No \\
\hline $\begin{array}{l}\text { Recurrencia de poliposis } \\
\text { nasal }\end{array}$ & No & Si (grado II) \\
\hline Control del asma & Sí & Sí \\
\hline
\end{tabular}


Cuadro 3. Esquema de desensibilización a ácido acetilsalicílico

\begin{tabular}{lccc}
\hline Día 0 & Día 1 & Día 2 & $\begin{array}{c}\text { Dosis de } \\
\text { mantenimiento }\end{array}$ \\
\hline $\begin{array}{l}\text { Placebo } \\
\text { Placebo }\end{array}$ & $\begin{array}{c}0.3 \mathrm{mg} \\
\text { Placebo }\end{array}$ & $300 \mathrm{mg}$ & $\begin{array}{c}300 \mathrm{mg} / \text { día } \\
\text { Posterior descenso } \\
\text { a } 150 \mathrm{mg} / \text { día. }\end{array}$ \\
Placebo & $150 \mathrm{mg}$ & \\
& $300 \mathrm{mg}$ & \\
& $\begin{array}{c}\text { Dosis acumula- } \\
\text { da: } 483.3 \mathrm{mg}\end{array}$ & \\
\hline
\end{tabular}

hasta alcanzar una dosis total acumulada de $483.3 \mathrm{mg}$. A los 30 minutos siguientes a la última dosis tuvo rinorrea y obstrucción nasal, reversibilidad con antihistamínico de primera generación y permaneción en vigilancia.

- Día 2: se administraron 300 mg de aspirina sin reacciones adversas. El procedimiento concluyó con desensibilización exitosa (Cuadro 1).

Se continuó con la administración de 300 mg al día como dosis de mantenimiento de forma ambulatoria, además de protección de la mucosa gástrica con un inhibidor de la bomba de protones, sin efectos adversos asociados con la ingesta de aspirina.

Se disminuyó la dosis a $150 \mathrm{mg}$ al día a los 3 años de la desensibilización y en la actualidad, después de trascurridos 4 años de la desensibilización, tiene buen control del asma con paso 2 de GINA, sin exacerbaciones (Asthma Control Test 24 puntos y FEV1 84\%) y control de los síntomas nasales sin necesidad de polipectomías, con recuperación parcial del olfato, sin otro tratamiento farmacológico. Al momento sin pólipos nasales.

\section{Caso 2}

Mujer de 49 años de edad, con antecedentes heredofamiliares de atopia, refiere anafilaxia asociada con la aspirina y metamizol, eritema polimorfo por penicilinas y anafilaxia por sulfas. A los 29 años de edad inició con rinosinusitis crónica moderada-severa persistente con poliposis nasal y anosmia y asma grave persistente, tratamiento con dosis altas de esteroide inhalado, LABA, inhibidores de los receptores de leucotrienos, esteroide nasal y ciclos cortos de prednisona a dosis de $1 \mathrm{mg} / \mathrm{kg} /$ día de forma frecuente, con múltiples exacerbaciones y pobre control; requirió 6 polipectomías nasales durante los últimos 10 años (1 polipectomía cada 1.6 años). Hace 10 años se le realizaron pruebas epicutáneas con aeroalergenos con resultado positivo a ácaros y pólenes de árboles, recibió inmunoterapia específica durante 3 años con escasa mejoría clínica.

Se aplicó el esquema de desensibilización descrito en el caso 1. Las complicaciones al tratamiento se observaron de la siguiente forma:

Día 1: rinorrea, obstrucción nasal, hiperemia y prurito ocular a los 20 minutos siguientes a la última dosis, síntomas que remitieron con antihistamínico de primera generación y esteroide, permaneció en vigilancia.

El tratamiento concluyó exitosamente, con dosis de mantenimiento de $300 \mathrm{mg}$ al día, con reducción a $150 \mathrm{mg}$ al día a los tres años de haber realizado la desensibilización, siguió asintomática, con paso 2 de GINA y esteroide nasal.

Durante los últimos cuatro años no ha tenido exacerbaciones de asma (Asthma Control Test 25 puntos y FEV1 87\%) con síntomas intermitentes de la rinosinusitis crónica, pólipos nasales grado Il bilateral en la endoscopia nasal, ni necesidad de requerir polipectomía y sin efectos adversos asociados con la ingesta de ácido acetilsalicílico.

\section{DISCUSIÓN}

El tratamiento de la enfermedad respiratoria exacerbada por aspirina incluye a los inhibido- 
res de los receptores de leucotrienos, esteroide intranasal, polipectomías, control del asma según su severidad y evitar la ingestión de antiinflamatorios no esteroides. En caso de requerir analgésicos se recomiendan los salicilatos no acetilados, por su inhibición débil de la COX1, como el salsalato, dosis bajas de paracetamol (máximo 1000 mg/día) o inhibidores selectivos de la COX2.

Existen algunos estudios en donde se ha indicado omalizumab y mepolizumab con buenos resultados. El hecho de no ingerir antiinflamatorios no esteroides no implica la desaparición de la enfermedad. ${ }^{6,7}$ Existen indicaciones para realizar la desensibilización al ácido acetilsalicílico, como en:

- Pacientes con poliposis nasal recurrente a pesar de un tratamiento farmacológico apropiado.

- Pacientes que requieran frecuentemente antiinflamatorios no esteroides, con evaluación del riesgo-beneficio.

La dosis indicada para mantenimiento de aspirina es de $325 \mathrm{mg}$ dos veces al día o $650 \mathrm{mg}$ dos veces al día, mientras que para pacientes con cardiopatía isquémica es de $81 \mathrm{mg}$ al día.

La desensibilización debe efectuarla el personal calificado y con experiencia; está contraindicada en pacientes con padecimientos pulmonares o cardiovasculares no estables o que muestren FEV1 menor de 70\%.,

Aunque algunos protocolos recomiendan iniciar con dosis de $81 \mathrm{mg}$, algunos autores señalan que $75 \%$ experimenta reacciones con dosis de 45 a $60 \mathrm{mg}$, por lo que se recomienda iniciar con 40.5 mg y duplicar la dosis cada 90 a 240 minutos. La mayoría de los autores recomienda intervalos de 3 horas, puesto que un alto porcentaje tiene reacciones a las dos horas de su administración.
En pacientes con reacciones severas, incluida la anafilaxia asociada con la ingesta de aspirina, se recomienda iniciar con dosis de $10 \mathrm{mg}$ o menos. La dosis de mantenimiento recomendada en la mayoría de los casos es de $650 \mathrm{mg}$ dos veces al día y reevaluar la eficacia a los tres meses si ha reportado beneficio se deberá continuar, de lo contrario se suspende. ${ }^{8,9}$

Los mecanismos implicados en la desensibilización a la aspirina sugieren que se induce un estado de tolerancia temporal a los antiinflamatorios no esteroides con reducción de la expresión de IL-4 y de la activación de STAT- $6 .{ }^{10}$

En caso de suspender la terapia en menos de tres días puede reiniciarse a la misma dosis; si el paciente suspende el tratamiento de 3 a 5 días debe reintroducirse a dosis de 325 mg con evaluación de la tolerancia; sin embargo, si se suspende más de 5 días se considera pérdida de la tolerancia. ${ }^{10}$

Los beneficios demostrados con la desensibilización a la aspirina incluyen la reducción de: síntomas de rinosinusitis crónica, número de intervenciones quirúrgicas debidas a la poliposis nasal, gravedad del asma, con dosis de $650 \mathrm{mg}$ cada $12 \mathrm{~h}$. Algunos pacientes también muestran estas ventajas con dosis de $325 \mathrm{mg}$ cada $12 \mathrm{~h}$, en menor proporción se exacacerban al reducir la dosis, la que debe incrementarse nuevamente. ${ }^{11}$

La principal causa de abandono del tratamiento es la toxicidad gastrointestinal asociada con la ingesta de ácido acetilsalicílico, por lo que se recomienda la prescripción concomitante de protectores de la mucosa gástrica. ${ }^{11}$

La enfermedad respiratoria exacerbada por aspirina afecta la calidad de vida de los pacientes y genera un alto costo del tratamiento farmacológico y quirúrgico; por esto se sugiere evaluar la utilidad del protocolo de desensibilización al ácido acetilsalicílico. 
En el caso 1 la paciente tenía el antecedente de 12 polipectomías y pobre control a pesar del paso 4 de GINA e inhibidores de los receptores de leucotrienos y esteroide intranasal. En la actualidad, luego de cuatro años de desensibilización al ácido acetilsalicílico se ha reducido la necesidad del uso de fármacos para el control del asma sin que haya tenido exacerbaciones. Con base en la evolución de la paciente puede determinarse que de no haber efectuado el protocolo de desensibilización, teóricamente le habrían realizado 3.6 polipectomías en ese periodo. Mostró alta efectividad en el control de la rinosinusitis crónica y la poliposis nasal y buena respuesta al control del asma.

Por lo que se refiere al caso 2, también se demostró alta eficacia del control del asma y la rinosinusitis crónica; en teoría, se calcula que de no haber realizado el protocolo de desensibilización habría requerido 2.5 polipectomías en los últimos 4 años.

En ambos casos la dosis de mantenimiento actual es de $150 \mathrm{mg}$ al día, a diferencia de lo que se señala en la bibliografía que sugiere mantener, al menos, la dosis de $325 \mathrm{mg}$ al día; manteniendo la efectividad y sin experimentar efectos adversos gastrointestinales.

\section{CONCLUSIÓN}

La desensibilización al ácido acetilsalicílico es un tratamiento útil en la reducción de los síntomas asmáticos y respiratorios, principalmente de rinosinusitis crónica y poliposis nasal. Además de disminuir la severidad del asma mejora la calidad de vida de los pacientes y reduce el costo del tratamiento farmacológico y quirúrgico. En ambos casos se demostró la eficacia de la desensibilización a la aspirina puesto que en los últimos cuatro años no han requerido ingresos al servicio de urgencias por exacerbaciones, con menor dosis de fármacos y sin necesidad de intervención quirúrgica. La dosis de mantenimiento se deberá ajustar individualmente, según la respuesta de cada paciente.

\section{REFERENCIAS}

1. Marek L. Kowalski, Classification of Reactions to Nonsteroidal Antiinflammatory Drugs. Immunol Allergy Clin N Am 2013, 33:135-145.

2. Laidlaw, T. Aspirin- Exacerbated Respiratory Disease. Up To Date, 2015; 1-20.

3. Rajan JP. Prevalence of aspirin-exacerbated respiratory disease among asthmatic patients: A meta-analysis of the literature. J Allergy Clin Immunol 2015; 135:676.

4. Laidlaw, T. Pathogenesis of Aspirin-Exacerbated Respiratory Disease and Reactions. Immunol Allergy Clin N Am. 2013:33;195-210.

5. Bochenek, G. Aspirin-Exacerbated Respiratory Disease: Clinical Disease and Diagnosis. Immunol Allergy Clin N Am, 2013, 33:147-161

6. Simon, R. Aspirin-Exacerbated Respiratory Disease: nSAID Challenge And Desensitization. Up To Date, 2014; 1-14.

7. Diario Oficial IMSS, 2015: 1-4.

8. Alvarez-Puebla, M. Inhibidores de la COX-2 en la enfermedad respiratoria exacerbada por aspirina. Alergol Inmunol Clin 2004; 19: 39-40

9. Lee J. Selection of aspirin dosages for aspirin desensitization treatment in patients with aspirin-exacerbated respiratory disease. J Allergy Clin Immunol 2007; 119:157.

10. Świerczyńska-K. Aspirin desensitization in patients with aspirin-induced and aspirin-tolerant asthma: a doubleblind study. J Allergy Clin Immunol 2014; 134:883.

11. Cho KS, Soudry E, Psaltis AJ, et al. Long-term sinonasal outcomes of aspirin desensitization in aspirin exacerbated respiratory disease. Otolaryngol Head Neck Surg 2014; 151:575. 